Universidad Nacional de La Plata.

Facultad de Humanidades y Ciencias de la Educación.

Departamento de Educación Física

\title{
Malestares y resistencias frente a las interpelaciones de la Educación Sexual Integral. Un análisis del posicionamiento de docentes de educación física
}

\author{
Discomfort and resistances to the challenges of \\ Comprehensive Sex Education. An analysis of the positioning \\ of physical education teachers \\ Jorgelina Marozzi \\ jmarozzi@upc.edu.ar \\ Universidad Provincial de Córdoba, Argentina \\ Facundo Boccardi \\ facundoccardi@gmail.com \\ Universidad Nacional de Córdoba, Argentina \\ Andrea Raviolo \\ andrearaviolo67@gmail.com \\ Ministerio de Educación de la provincia de Córdoba, Argentina
}

Recepción: 28 Mayo 2020

Aprobación: 03 Septiembre 2020

Publicación: 09 Octubre 2020

Cita sugerida: Marozzi, J., Boccardi, F. y Raviolo, A. (2020). Malestares y resistencias frente a las interpelaciones de la Educación Sexual Integral. Un análisis del posicionamiento de docentes de educación física. Educación Física y Ciencia, 22(4), e150. https:// doi.org/10.24215/23142561e150
Resumen: En el área de la Educación Física (EF), la propuesta de la Educación Sexual Integral (ESI) problematiza la división tradicional por género e interpela prácticas educativas tradicionales, fuertemente institucionalizadas, en pos de incluir diferentes lenguajes creativos y expresivos que promuevan una diversidad de prácticas corporales. El siguiente trabajo presenta resultados de una investigación desarrollada en Córdoba que indagó las resistencias docentes frente a la implementación de la ESI. Se analizaron materiales discursivos recolectados en discusiones colectivas entre docentes de EF producidas en el marco de capacitaciones y en entrevistas individuales. En términos generales, las resistencias en torno a la clase mixta responden a la percepción de diferencias significativas entre varones y mujeres, la contradicción entre el deporte escolar mixto y el deporte hegemónico, la dificultad para elaborar construcciones didácticas y la lectura erótica del lenguaje y comunicación corporal de los/as estudiantes. Dichas resistencias, en la mayoría de los casos, evidencian desconocimiento del enfoque y modo de abordaje de la ESI. El estudio concluye con la necesidad de desmontar los supuestos del profesorado acerca del cuerpo, el género y el sentido de la EF en la escuela; y avanzar en capacitaciones sobre enfoques críticos que abran este espacio curricular a la propuesta de la ESI.

Palabras clave: Educación sexual, Educación física, Coeducación, Práctica educativa, Deporte.

Abstract: In the area of Physical Education (PE), the proposal of Comprehensive Sex Education (CSE) questions the traditional gender division and challenges strongly institutionalized traditional educational practices, in order to include different creative and expressive languages that promote a diversity of body practices. he following work presents the results of an investigation carried out in Córdoba that investigated the teaching resistance against the implementation of the CSE. Discursive materials collected in collective discussions between PE teachers produced in the framework of training and in individual interviews were analyzed. In general, the resistance around the mixed class responds to the perception of significant differences between men and women, the contradiction between mixed school sport and hegemonic sport, the difficulty in developing didactic constructions and erotic reading of language and students' body communication. hese resistances, in most cases, show lack of knowledge about the approach 
to CSE. The study concludes with the need to dismantle teachers' assumptions about the body, gender and the meaning of PE in school, and advance in training on critical approaches that open this curricular space to the CSE proposal.

Keywords: Sex education, Physical education, Coeducation, Educational practice, Sport.

\section{Introducción}

La implementación de la Educación Sexual Integral (ESI) en Argentina constituye un proceso complejo ya que interpela roles y prácticas educativas arraigadas en las instituciones escolares. Desde comienzos de este siglo, la necesidad de políticas públicas que promuevan la educación sexual en las escuelas se ha instalado progresivamente en toda la región en consonancia con las directrices de organismos internacionales y las demandas de movimientos de mujeres (Báez, 2016; Boccardi, 2020). Este proceso ha configurado un espacio de confrontaciones entre diferentes actores y posicionamientos que adquirió particularidades e intensidades específicas en cada uno de los países pero que en todos los casos actualizó viejas disputas acerca de la sexualidad y la intervención estatal. En Argentina, los debates legislativos que precedieron a la sanción de la ley se trasladaron a la agenda mediática donde se trabaron disputas acerca del rol del Estado en la definición de los contenidos de la educación sexual, los alcances y las injerencias de las familias, las edades adecuadas de las/os estudiantes y la idoneidad de las/os docentes para desempeñar esta práctica educativa (Boccardi, 2010). La sanción de la Ley $\mathrm{N}^{\circ} 26.150$ en octubre de 2006 produjo dos hitos fundamentales que desencadenaron los procesos de implementación que aún atravesamos: uno de ellos consistió en establecer la garantía del derecho y la responsabilidad estatal referida a la sexualidad de las/os educandas/os y el otro hito fue la formulación del enfoque integral para superar las perspectivas reduccionistas de la sexualidad provenientes de las tradiciones moralizantes y biologicistas. Este enfoque aparece anticipado en el texto de la ley y encuentra su carnadura conceptual y pedagógica en los Lineamientos Curriculares para la ESI publicados en el 2008 y en la sucesiva serie de materiales didácticos producidos por el Programa Nacional de ESI. La noción de integralidad ocupa un lugar fundacional en la propuesta oficial de la ESI, ya que le atribuye una cualidad superadora de tradiciones precedentes que o bien limitaban la sexualidad a la genitalidad y la abordaban desde la potencialidad de sus riesgos, o bien la sometían a regulaciones de orden moral (Boccardi, 2014). En ese sentido, el carácter integral de esta política educativa propone el abordaje de la sexualidad a partir de una articulación entre perspectivas referidas a la afectividad, los derechos, la diversidad sexual, el género y el cuidado de la salud que permiten superar las reducciones genitalistas y, a su vez, tranversalizarla en toda la currícula de los diferentes niveles del sistema educativo.

El enfoque integral de la sexualidad que sostiene esta política impele a las escuelas, fundamentalmente, a desmontar los mecanismos de discriminación y exclusión sustentados en estructuras patriarcales 
(Millet, 1975) y heteronormativas (Warner, 1991) fuertemente institucionalizadas que producen desigualdades y legitiman opresiones. En esta operatoria, el rol de las/os docentes aparece como uno de los aspectos centrales. Por esa razón, el proceso de implementación de esta política pública ha privilegiado estratégicamente la capacitación y actualización de las/os educadoras/es como punto de partida.

Por otro lado, esta perspectiva desplegada por la ESI le otorga un peso significativo a las regulaciones corporales que tienen lugar en la escuela. En ese sentido, dada la centralidad de la dimensión corporal, el espacio curricular de Educación Física (EF) atrae la atención de los dispositivos diseñados para la implementación de la ESI.

La escuela y la Educación Física contribuyó a la diferenciación modelando los cuerpos, prescribiendo actividades físicas y deportivas para cada sexo. De acuerdo con ello, la literatura especializada coincide en señalar la persistencia de una configuración de la clase de EF tendiente a reproducir las normas de género hegemónicas. Diversos estudios producidos en diferentes países del mundo, y especialmente en España (López Estevez, 2012; Sanchez Perez, 2012; Serra Payeras, 2016) como en nuestro país (Scharagrodsky, 2006; Fernández, 2011, Di Gregorio, 2013, Kopelovich, 2017; Pellegrini Malpiedi, 2015) muestran que en la Educación Física escolar se reproducen estereotipos tradicionales de género relacionados con la actividad física y el deporte, como el privilegio en el uso de tiempos, espacios y materiales de parte de los varones, la actividad y expansividad corporal de éstos en contraposición con la pasividad de las mujeres, a cuyos movimientos se le atribuye el carácter de negativo o ridículo. Estos estereotipos funcionan como obstáculos o limitaciones para que se desarrollen procesos coeducativos en este ámbito. Otros estudios indagan específicamente acerca de concepciones o representaciones de las/os docentes de educación física que contribuyen a la reproducción de estereotipos y prácticas sexistas. Así, Scharagrodsky (2006) plantea que las relaciones de poder entre varones y mujeres que se configuran en las clases de educación física están avaladas por las representaciones y actividades propuestas por las/os docentes, que con sus supuestos sobre la masculinidad y la feminidad institucionalizan ciertas normas, prácticas y prioridades para cada género, contribuyendo a la reproducción de desigualdad.

Si bien son numerosos los trabajos que indagan acerca de las propuestas de EF a la luz de los aportes de género, no ocurre lo mismo con la recepción del enfoque de la ESI. Debido a que se trata de una política reciente, las investigaciones que avanzan en caracterizar la recepción de este enfoque en el colectivo docente y su efectiva implementación se están produciendo actualmente.

Los Lineamientos Curriculares para la Educación Sexual Integral (2008) y los materiales didácticos oficiales problematizan la división por género arraigada tradicionalmente en las propuestas de $\mathrm{EF}$, y promueven la clase mixta y el desarrollo de prácticas inclusivas. Además, instan a la revisión de prácticas centradas en el aprendizaje de deportes para 
incluir diferentes lenguajes expresivos y creativos, en distintos formatos y propuestas que promuevan una diversidad de prácticas corporales.

En relación a la conformación de agrupamientos mixtos, investigaciones en la última década evidencian desacuerdo de parte del cuerpo docente. A partir de una investigación desarrollada en La Matanza (Buenos Aires, Argentina), Gómez y Tevez (2013) refieren que el profesorado sustenta su oposición a la implementación de la clase mixta en dos argumentos principales: la existencia de diferencias biológicas entre varones y mujeres; y la dificultad de alcanzar un óptimo rendimiento deportivo. Aseguran además que cuando estos argumentos se desactivan, al revisarlos a la luz de enfoques constructivistas y humanistas de la Educación Física, aparecen otros argumentos: la peligrosidad que representan los cuerpos en contacto, y la necesidad de sostener los proyectos que vienen realizando. Por otra parte, Di Gregorio (2013), en una investigación realizada en un distrito de la provincia de Buenos Aires, relevó que casi el 70\% de las/os docentes del estudio no estaba de acuerdo con la implementación de clases mixtas en el nivel secundario. Los principales argumentos sostenidos por estas/os docentes giraban en torno a la existencia de diferencias físicas determinantes entre ambos sexos $\mathrm{y}$ a los diferentes intereses y actitudes en relación a la materia. La autora identificó además que la concepción de EF que subyace continúa anclada en el paradigma racional positivista. En coherencia con ello, prevalece una fundamentación biologicista de las diferencias entre varones y mujeres. Además, en las prácticas observadas de estas/os docentes se evidencia una apuesta a lo deportivo profesional que aspira a preparar a las/os alumnas/ os para un tipo y grado de competencia deportiva establecido por el discurso del deporte de alto rendimiento.

En la provincia de Córdoba, la Subdirección de Educación Física del Ministerio de Educación de la provincia, en el año 2015 emitió una disposición que instó a las escuelas a abandonar la tradicional división por sexos y a constituir grupos integrados por todas/os las/ os estudiantes de cada sección o año. Dicha disposición fue canalizada por algunas de las zonas de inspección, y generó en el cuerpo docente diferentes reacciones a favor y en contra de cumplir con esta medida. Los desacuerdos, expresados en diferentes instancias de formación docente organizadas por el Programa de ESI, dieron origen a la necesidad de una indagación más sistemática que permitiera conocer con mayor detalle la existencia de obstáculos que inciden en la efectiva implementación de esta política pública. Por este motivo, se hizo necesario generar conocimiento prestando especial atención a las resistencias que se instalan, ya que configuran un posicionamiento docente particular que obstaculiza el avance de la ESI en este espacio curricular. Tal fue el objetivo de la investigación que se desarrolló en la provincia de Córdoba en los años 2017-2019 por un equipo conformado por integrantes del Programa de ESI y docentes de la Universidad Provincial de Córdoba cuyos resultados se presentan en este artículo. 


\section{Metodología}

Para recoger los discursos de docentes de Educación Física que sostienen la resistencia o rechazo ante la implementación de la ESI, se llevaron a cabo dos estrategias. En un primer momento se recogió información acerca de estas resistencias en las discusiones que se generaron entre docentes de educación física que participaron de un dispositivo de capacitación docente acerca de la implementación de la ESI en la clase de educación física. Se llevaron a cabo tres cursos de capacitación coordinados por el Programa de Educación Sexual Integral junto al Área Curricular de Educación Física del Ministerio de Educación de la provincia de Córdoba, a los cuales asistieron 130 docentes, quienes optaron de manera voluntaria por esta oferta formativa. Cada curso constó de dos encuentros, en los cuales se plantearon consignas que proponían a las/os docentes asumir y explicitar y argumentar su posicionamiento en diferentes temáticas vinculadas a la ESI.

En un segundo momento, y para profundizar en este modo particular de posicionamiento, se realizaron entrevistas a siete docentes que se desempeñaban en el nivel secundario, en diferentes escuelas de la provincia de Córdoba, tanto de gestión estatal como de gestión privada, y que habían manifestado públicamente su oposición a las modificaciones organizacionales y didácticas que propone la ESI, a través de notas presentadas a directivos de sus escuelas e inspectoras/es. Las/os entrevistadas/os se incluyeron progresivamente hasta lograr la saturación de las categorías. De esta manera se utilizó un muestreo teórico (Glaser \& Strauss, 1967) ya que la selección de los participantes se realizó siguiendo criterios fundamentalmente teóricos que se fueron adoptando en el curso del trabajo de campo.

Es importante tener en cuenta tal como proponen diversos autores (Giordan y De Vecchi, 1988; Pozo, Del Puy Pérez, Sanz y Limón, 1992) que los supuestos docentes que se pretenden indagar no se identifican solamente con lo que un sujeto dice cuando se le pregunta sobre algún asunto, sino que corresponden a estructuras o sistemas de significados que se infieren a partir de distintas expresiones del sujeto. Esta particularidad complejizó los procesos de análisis de la información, obligando a las/os investigadoras/es a sondear regularidades subyacentes, sentidos implícitos, y no sólo tomar los conocimientos explícitos que las/ os entrevistadas/os pueden verbalizar con facilidad.

El corpus discursivo fue analizado a través de procesos de codificación y se construyeron categorías para dar cuenta de los argumentos que sostiene el profesorado.

\section{Resultados y discusión}

Las resistencias en torno a la implementación de la ESI se concentran en torno a dos aspectos: 
1. Resistencias a la modificación de los agrupamientos tradicionales por sexo a agrupamientos mixtos.

2. Obstáculos referidos a las modificaciones en la enseñanza que propone la ESI

Se presenta a continuación los resultados del análisis de cada una de estos tópicos, y las categorías construidas.

\subsection{Resistencias a la modificación de los agrupamientos tradicionales por sexo a agrupamientos mixtos}

Este obstáculo cobra gran relevancia. La principal oposición a la implementación de la ESI se concentra en modificar los agrupamientos de manera tal de trabajar conjuntamente varones y mujeres. Categorizamos las resistencias de las/os docentes según se relacionen o estén vinculadas a:

- Las diferencias entre varones y mujeres.

La percepción de las diferencias individuales de manera binaria (varones-mujeres) es el núcleo de mayor resistencia para la organización de los agrupamientos de manera mixta. Estas diferencias se presentan en torno a dos órdenes: por un lado remiten a las diferencias en capacidades físicas: "Biológicamente son diferentes" (Andrea, 52), "porque hay diferencias sexuales, más se da en la parte adolescente, la velocidad, las potencialidades son distintas. Hay diferencias de crecimiento" (Graciela, 55). Por otro lado, a las diferencias actitudinales: "Los varones necesitan descargar su impronta masculina" (Andrea, 52), "Los varones se mueven más rápido, entonces hacen más dinámico el juego" (...) "Las chicas más organizadoras -en vida en la naturaleza- en los juegos los chicos son más dinámicos, más predispuestos, nunca tienen una queja para hacer una actividad" (Graciela, 55), "En voley tengo tres mujeres y tres varones, las chicas están paradas, no van a hacer un desplazamiento" (Mario, 48), "A las chicas las tenés que pinchar todo el tiempo para que hagan algo que las entusiasme, mientras que a los varones les tiras una pelota y se ponen a jugar" (Grupo 3).

En estos argumentos, podemos inteligir que funciona con fuerza un modo binario de leer las diferencias, las cuales no están siendo consideradas desde las particularidades de cada sujeto, sino generizadas: los/as estudiantes asumirían ciertas características sólo por pertenecer a un determinado colectivo (varones-mujeres). Los cuerpos son leídos de manera dicotómica, y las características diferenciales formarían parte de la esencia de cada colectivo. Funciona así un esencialismo biológico (Vance, 1997) ya que sería la anatomía de los cuerpos definida al nacer la que llevaría al desarrollo de capacidades físicas diferentes para ambos sexos. Aún cuando pudieran percibir que muchas diferencias son actitudinales, producto de una construcción 
corporal atravesada por la historia del sujeto, se les dificulta pensar en la posibilidad de transformación de estas actitudes.

$\mathrm{Si}$ profundizamos el análisis de los enunciados docentes, observamos que las diferencias identificadas están valoradas de modo desigual. Algunas afirmaciones sostienen que "las capacidades masculinas son superiores" (Grupo 1). En relación a la feminidad y masculinidad, se invisibiliza el proceso de construcción, y se mantiene la jerarquización de lo masculino, en tanto lo femenino se mide en función del parámetro o desempeño motriz masculino que es lo que se considera correcto o normal. Esto es coincidente con diversos resultados de estudios en la temática (Scharagrodsky, 2004; Kopelovich, 2017; Galak, 2009).

Desde esta visión, trabajar conjuntamente traería aparejado consecuencias negativas para ambos colectivos: en las mujeres la desmotivación, ya que no se expondrán corporalmente por temor a ser golpeadas, lo cual a su vez disminuiría su autoestima, y en los varones afectaría el desempeño ya que tendrían que limitar su potencial para no golpear a las chicas. Lo cual también generaría desmotivación. Varios enunciados refieren esto: "se limitan, no hacen nada, porque tienen vergüenza de que les digan algo o aunque no les digan nada se sienten incómodas" (Grupo 3), "Con la clase mixta hay más deserción, los niveles de ejecución y rendimiento cambiaron, los chicos están desmotivados" (Grupo 2), "En dos de los grupos tuve mucha deserción de mujeres, por no querer hacer clases con los varones" (Claudia, 42).

Las mayores dificultades se concentran en la práctica de deportes. Teniendo en cuenta la centralidad que ocupa la enseñanza de deportes en las clases de $\mathrm{EF}^{3}$, cada vez que las/os docentes manifiestan desacuerdo con las clases mixtas, están suponiendo una clase deportiva, desde una concepción tradicional de la EF en la que prevalece el sentido de rendimiento físico, eficacia y eficiencia en los movimientos técnicos desde un modelo conductista.

- Reconocimiento de la contradicción entre el deporte escolar mixto y el deporte hegemónico.

Las/os docentes que sostienen la resistencia a las clases mixtas aluden como argumento que las ligas o campeonatos que ofrecen las agencias provinciales o entidades nacionales para que participen las escuelas no son mixtos. Las/os docentes explicitan esta contradicción como justificativo para sostener clases separadas al interior de su asignatura. En el caso en que se trabaje el deporte escolar en forma mixta, no se desarrollan las habilidades necesarias para competir en eventos deportivos fuera de la escuela. En coherencia con ello, una docente plantea:

Dificulta todo lo que yo quiera alcanzar, si yo quiero que aprendan los conocimientos básicos del deporte, entienda la táctica del deporte y más o menos lo juegue reglamentariamente, yo puedo trabajarlo mixto, lo que no voy a poder es sacar un equipo ganador. (Graciela, 55) 
Claramente se observa que el objetivo de la enseñanza está puesto en el resultado, en la competencia, desde un enfoque deportivo de la EF. Lejos de aprovechar la potencialidad educativa del deporte en tanto práctica capaz de provocar aprendizajes necesarios para el desarrollo corporal, expresivo, motriz, y suscitar reflexiones y valoraciones, la EF se convierte en la transmisión de contradicciones sociales que el deporte de competencia reproduce:"existencia de jerarquías (a temprana edad), ámbito de discriminación (los más talentosos son mejor atendidos), desigualdades de género, exclusión en lugar de inclusión (trabajar solo con los que juegan en el equipo escolar) exigencias físicas y psicológicas desmedidas para los sujetos" (Gómez, 2009 p. 221).

Participar de certámenes deportivos extraescolares implica asumir los códigos de la institución deportiva. Los certámenes asumen un carácter orientador y normativo para el trabajo de las/os profesoras/es de EF en las escuelas. Tendríamos entonces, como sostiene Valter Bracht (1996) "no el deporte de la escuela y si el deporte en la escuela, lo que indica su subordinación a los códigos/sentidos de la institución deportiva” (p.23).

Estas lógicas de funcionamiento del campo deportivo no serían compatibles con las lógicas que rigen el campo educativo donde se desarrolla la EF. En el campo deportivo, el sentido competitivo, selectivo, exclusivo, en búsqueda de resultado prevalece, y esa característica trasladada al ámbito escolar que pretende igualdad, solidaridad, cooperación, que indaga procesos, produce una tensión que desvanece los objetivos de la educación. Tal como lo expresa la Ley de Educación Nacional en su Art. 8, "La educación brindará las oportunidades necesarias para desarrollar y fortalecer la formación integral (...) y promover en cada educando/a la capacidad de definir su proyecto de vida, basado en los valores de libertad, paz, solidaridad, igualdad, respeto a la diversidad, justicia, responsabilidad y bien común" (Ley 26.206, 2006)

Por otra parte, varias/os docentes explicitaron cierta presión del equipo directivo para resaltar el centro educativo a través del deporte. Participar y "ganar" aumenta el estatus de la institución. También subyace la idea que los triunfos deportivos ponen en valor al docente de $\mathrm{EF}$, ubicándose en una jerarquización según los logros. Esto es coincidente con la idea de docente-entrenador cuya función es "cazar talentos" al descubrir a las/os estudiantes más hábiles, que se destacan en lo deportivo. Así, el éxito o fracaso de parte de las/os estudiantes es un signo del éxito o fracaso del profesor/a y/o de la institución.

Reconociendo las situaciones de exclusión que se generan en el armado de equipos para jugar, algunas/os docentes sostienen la creencia que pasar por situaciones de exclusión "los hace más fuertes" (Grupo 1) las/os prepara para una sociedad competitiva. Aquí subyace la idea de que la escuela para que la/el estudiante se 
adapte a la sociedad, y no que la escuela promueve propuestas que aporten a la transformación de prácticas sociales.

- Dificultad en las construcciones metodológicas para las clases mixtas.

Las/os docentes refieren "no saber" cómo trabajar en forma mixta, no contar con estrategias para ello. De su discurso se desprende que, quienes atravesaron un proceso de transformación de clases separadas por género a clases conjuntas, no necesariamente modificaron sus propuestas didácticas, sino que para algunas/os docentes implicó la extensión de las propuestas tradicionales a todo el estudiantado.

Algunos enunciados dan cuenta de las dificultades que se les presentan durante el proceso de enseñanza. Refieren: "Yo incluyo, pero si no quiere jugar al fútbol que juegue a la soga, al quemado, que se siente" (Andrea, 52), "La chica que quiere jugar se tiene que adecuar y aguantar y soportar" (Graciela, 55), "los varones quieren jugar al fútbol, y algunas chicas se adaptan, pero otras no y se apartan" (Mario, 48). Vemos en estos enunciados que son "las chicas" las que deben adaptarse a una propuesta tradicionalmente pensada para varones. "La utilización del modelo androcéntrico, que suele identificarse con el modelo deportivo, supone una discriminación para la mujer, al no coincidir con el estereotipo tradicional femenino" (Vázquez y Gómez, et al, 1990, p. 13) Así, las construcciones metodológicas que implementan las/os docentes no tienen en cuenta la reconfiguración de los grupos que el contexto actual presenta.

Si bien no era un objetivo de la investigación indagar acerca de la relación entre la formación recibida y las dificultades a la hora de construir una propuesta coeducativa, los enunciados nos permiten hipotetizar que dicha formación estuvo marcada por enfoques tecnicistas y biologicistas de la EF (Bracht y Crisorio, 2003), con un predominio de la enseñanza de la técnica de los deportes. Si la formación estuvo marcada por el aprendizaje y la práctica de deportes hegemónicos, donde aprender implica automatizar una técnica para alcanzar los mejores niveles de eficacia deportiva, en consecuencia, el énfasis está puesto en las diferencias físicas, y las particularidades del desarrollo biológico diferenciado asumen mayor importancia que cualquier otro criterio a la hora de, por ejemplo, formar equipos para jugar. (Argentina, Ministerio de Educación, 2012) Esto es coincidente con los resultados de una investigación desarrollada por Trueba (2007) quien postuló que en el profesorado prima la creencia que el objetivo principal de la disciplina consiste en el desarrollo de las capacidades condicionales (fuerza, resistencia y velocidad) por sobre otros objetivos como son la integración, la recreación, la construcción de ciudadanía, etc. Desde el enfoque actual de la ESI, en estas prácticas se invisibiliza al cuerpo que siente, se emociona 
y se expresa quedando reducidas las posibilidades de incorporar diferentes propuestas expresivas, lúdicas, recreativas.

También se observó que algunas/os docentes realizan adaptaciones al trabajar con grupos mixtos. Algunas de ellas: "Les digo hoy estamos con chicas, no sean tan torpes, bajemos el nivel, bajemos la ansiedad (...) Tenemos que decir no hacen el gol si no se la pasan a una chica" (Graciela, 55), "los varones hacen 10 flexiones de brazos y las chicas 5, 6, con las rodillas apoyadas" (Mario, 48). Otro enunciado refiere:

Cuando jugamos por ejemplo un partido, los varones tiran con la mano inhábil al arco si hay una mujer, o sea con la izquierda, yo le planteo, una para que tiren menos fuerte (...) Si hay un arquero varón, el varón tira libre, tira con su habilidad y con la potencia que quiera tirar. (Marcelo, 46)

Las adaptaciones se realizan sobre las reglas de los juegos o deportes que se están enseñando o en la cantidad de ejercicios solicitados, y siempre implica limitar las habilidades masculinas, que se consideran superiores, y obligar o alentar a la participación femenina, ya que esta no estaría garantizada. La adaptación se hace conservando el mismo modelo de clase en el cual el énfasis está puesto en la adquisición de técnicas para aumentar el rendimiento. Estas estrategias también se apoyan en una concepción naturalista de los cuerpos, ya que se prescriben de antemano actividades diferenciadas a cada colectivo (mujeresvarones) sin tener en cuenta las posibilidades reales de cada uno. Los varones, por el solo hecho de serlo, deben limitar su capacidad o demostrar su resistencia haciendo más ejercicios. Nuevamente se observa que las diferencias entre los sujetos son leídas dicotómicamente, y en esa lectura funcionan estereotipos acerca de las capacidades y habilidades de las mujeres y varones.

- Lectura erótica del lenguaje y comunicación corporal.

En términos generales, la presencia de la dimensión erótica en la escuela siempre adquiere una carga negativa. Si bien la tradición iluminista fundacional que aún atraviesa el discurso pedagógico ha sido cuestionada y flexibilizada en las últimas décadas (Abramowski, 2012) desde posiciones que demandan una mayor atención a lo corporal y lo afectivo, lo erótico conserva un sentido negativo. La axiologización negativa del componente erótico al interior de la escuela, se halla imbricada en una extensa tradición de las sociedades occidentales modernas que le ha atribuido a la sexualidad adolescente y juvenil rasgos de transgresión y peligro (Margulis, 2003; Jones, 2010). Desde la sociología británica de los años setentas, se ha producido la categoría de pánico moral para analizar una serie de fenómenos culturales reactivos frente a comportamientos juveniles leídos como una amenaza al orden social (Thompson, 2014). Uno de los objetos centrales de dicho pánico fueron las conductas sexuales de la juventud que desde la década del sesenta en los países 
industrializados fueron configuradas como una amenaza a los valores tradicionales de la familia (Jenkins, 1998). Esta valoración de la sexualidad de jóvenes y adolescentes sedimenta un sustrato de sentidos cuyos rasgos vemos emerger en el discurso de algunas/ os docentes acerca de la dimensión erótica de la sociabilidad estudiantil en la escuela.

En el caso específico de clase de educación física en el nivel secundario, la presencia siempre amenazante y disruptiva del erotismo aparece, por un lado, en la interacción entre docentes y estudiantes y, por otro, en la interacción entre estudiantes. En ambos casos, se encuentra marcada genéricamente y regida por parámetros heterosexuales y cisexuales. Siguiendo la propuesta de Michael Warner (1991), entendemos que la función normativa de la heterosexualidad atraviesa las prácticas culturales, legales e institucionales instalando el supuesto de la existencia exclusiva de dos géneros dicotómicos determinados por el binarismo sexual biológico y estableciendo que la única atracción sexual natural y aceptable es la que se dirige hacia el sexo opuesto. En coherencia con esta formulación, articulamos necesariamente la noción de heteronormatividad a la de cisnormatividad (Serano, 2007), ya que bajo esta matriz normativa las identidades sexo-genéricas suponen siempre una coherencia con el sexo asignado al nacer.

En los argumentos que hemos recorrido acerca de la clase mixta de educación física, esta configuración de los agrupamientos es presentada como la condición de posibilidad de la emergencia de lo erótico. La propuesta de mezclar a varones y mujeres en un espacio curricular donde el cuerpo y el movimiento ocupan un lugar protagónico es vista por muchos/as docentes como una incitación peligrosa: "Meterse en clase mixta nos metemos en problemas que antes no teníamos" (Grupo 1)

Separar los sexos en educación física fue un mandato que no solamente afectó a la población estudiantil sino también a las/os docentes. En algunas jurisdicciones de manera explícita y formalizada (Vallarino, 2017) y en otras de manera implícita, la correspondencia entre la identidad sexual de estudiantes y docentes de educación física como condición para la enseñanza funcionó como una norma cuya vigencia ya casi se ha extinguido, aunque sus fundamentos aún gocen de una relativa legitimidad. En ese sentido, varias/os docentes le atribuyen a esa separación de los géneros un rasgo de resguardo y protección argumentando que en las condiciones actuales se "exponen a cuestiones legales más que antes" (Grupo 1). De acuerdo con ello, el fantasma de la denuncia de abuso sexual -y los abusos sexuales efectivos- se disiparían evitando que docentes y estudiantes de identidades sexuales opuestas se reúnan en la misma clase, ya que la matriz heteronormativa coloca a dicha oposición de los sexos como condición de posibilidad del contacto sexual. 
Por otro lado, en el caso de la configuración de los agrupamientos, si bien se comparte el mismo presupuesto heteronormativo, los argumentos difieren. En este caso, el eje aglutinador no es el riesgo del abuso sexual, sino más bien la fluctuación entre el desborde y la vergüenza. Reunir a varones y a mujeres en el desempeño de prácticas educativas que implican poner el cuerpo en movimiento y en eventuales situaciones de contacto, aparece en el discurso de muchas/os docentes de educación física como un factor que perturba el desarrollo de la clase promoviendo la incomodidad, principalmente, de las estudiantes. En esa línea argumentativa, algunas/os docentes señalan que la seducción y la vergüenza operan como obstáculos en la participación de las estudiantes en las clases de educación física cuyos efectos van desde la negativa a participar hasta el abandono de la materia. Esta situación es ubicada en un momento específico del desarrollo evolutivo de las estudiantes que vinculan al interés por seducir a los varones cuyos efectos van de la negativa a participar en los juegos colectivos a la deserción del espacio curricular: "cuando las chicas en tercer año empiezan a presumir, ya no quieren jugar" (Andrea, 52); “muchas mujeres no se animan a moverse frente a un compañero varón, entonces prefieren faltar a clases (...) porque la vergüenza puede más" (...) "ahora desaparecieron siete chicas cuando en la segunda parte [del año] empezaron a hacer juegos colectivos, en la primera parte trabajaban más el movimiento individual" (Claudia, 42)

De acuerdo con los argumentos expresados, el componente erótico es identificado invariablemente como un factor negativo que altera, limita u obstruye las posibilidades de la práctica educativa. Tal como vimos este obstáculo puede afectar tanto a la relación docente-estudiante como a las interacciones entre estudiantes. Esta lectura del erotismo se inscribe en una tradición discursiva que le atribuye a la sexualidad adolescente y juvenil componentes disruptivos que ponen en riesgo diferentes dimensiones del tejido social. La salida a estos problemas encuentra su modelo en la construcción de un tiempo pasado de la educación física donde los sexos no se mezclaban y, con ello, se eliminaba la interferencia erótica al desarrollo de la clase. En estos supuestos, la heterosexualidad aparece como la única configuración posible del erotismo que le atribuye sentidos específicos vinculados necesariamente al cortejo sexual e impide pensar otros tipos de tramitar la carga erótica de las interacciones educativas.

\subsection{Obstáculos referidos a las modificaciones en la enseñanza que propone la ESI}

En este espacio curricular, el enfoque integral de la ESI introduce la necesidad de revisar perspectivas vigentes en las prácticas concretas 
de enseñanza que replican lógicas deportivas competitivas y concepciones instrumentalistas del rendimiento físico. Con el cuestionamiento y desplazamiento de estas perspectivas, el enfoque integral se orienta a promover en las/os estudiantes la comunicación corporal con énfasis en el cuidado de sí misma/o y de otras/os, el despliegue de las propias posibilidades en un marco de igualdad de oportunidades, el respeto por la diversidad de identidades y posibilidades motrices, lúdicas y deportivas, la reflexión sobre la competencia y la promoción de prácticas cooperativas y solidarias (Argentina, Ministerio de Educación, 2008). Ante esto, visualizamos en las/os docentes participantes de este estudio un escaso conocimiento del enfoque y modo de abordaje de la ESI. A modo de ejemplo, se presentan dos modos de implementar la ESI que refieren dos docentes:

\footnotetext{
Digo cambios adolescentes a ver 'vengan los varones para acá'. Las chicas estaban jugando al hándbol, en ese momento los dividí porque tenía que hablar de sus cambios, entre ellos, no frente a las chicas en ese momento, entonces hablé con ellos mientras las chicas jugaban. Y después hice al revés, hablé con las chicas mientras los varones jugaban. Tuve que hacer esos malabares (Nélida, 56)

Se armó un cronograma que participaban profes de distintas áreas, a mí me tocó con historia y geografía armar un proyecto de ESI (...) Primero teníamos que hacer cada profe solo, entonces yo busqué dos videos de diversidad y discriminación que eran muy didácticos (...) tenían que traer un afiches, fibrones, y yo les tiraba una palabra a cada grupo, transexual, homosexual, bisexual, y cada grupito ponga un concepto de esa palabra, de lo que entienda, y después fuimos a ver el video (...) después tenían que traer una figura del diario, revista o dibujar lo que le había tocado y pegar en el afiche (Marcelo, 46)
}

El profesorado percibe a la ESI como un conjunto de contenidos cercanos a otros campos de conocimientos y alejado de la actividad corporal y motriz propia del campo de la educación física. Para las/ os docentes de este estudio, implementar la ESI implicaría "salirse" de la enseñanza de prácticas motrices y deportivas para abordar un tema específico, con metodologías propias de otros campos de conocimiento. Así, las actividades se reducen a "conversar" con las/os estudiantes acerca de temáticas relacionadas con la sexualidad, a la propuesta de búsqueda de información, exposiciones teóricas, o discusión en torno a videos o películas. No encontrar vinculación con las prácticas motrices propias de este campo obtura la posibilidad y la predisposición para transversalizar la ESI en las propuestas de enseñanza.

Por otra parte, en algunas/os docentes subyace la creencia de que con la mera reorganización grupal en clases mixtas ya se estaría implementando, aunque no se haga ninguna intervención pedagógica específica, lo que implica un desconocimiento de las intervenciones docentes necesarias para la enseñanza de la ESI. Refieren:

Trabajamos sin querer la ESI nada más que no lo ponemos en el libro de tema, si yo me pongo a trabajar con vos en vóley estoy haciendo una relación con vos, didáctica, de ensayo y error, de correcciones, y yo estoy aceptando que vos tenès limitaciones o yo tengo limitaciones. Por ahí tengo una chica que tiene mucha habilidad para el deporte y un varón que no, y bueno, se complementan, y hay una relación ahí (Claudia, 42) 
Cuando tengo que trabajar tomados de la mano, o sosteniendo, hacemos ejercicios con elementos bastón, soga entonces ese ejercicio de trabajar con el otro (Marcelo, 46)

Las intervenciones específicas que son requeridas para sostener un abordaje coeducativo, como es la actuación ante la reproducción de prácticas sexistas, y la atención específica para brindar real igualdad de oportunidades, son escasamente consideradas como prácticas docentes intencionales necesarias para transversalizar la ESI en las propuestas.

\section{Conclusiones}

La investigación realizada permitió reconocer dos ámbitos donde se concentra la resistencia a la implementación de la ESI: la modificación de los agrupamientos tradicionales por sexo a agrupamientos mixtos y la modificación de propuestas de enseñanza. El primer aspecto, la oposición a la implementación de clases mixtas a su vez está sostenido en cuatro argumentos que adquieren diferente peso en los discursos docentes: la consideración de las diferencias entre varones y mujeres, el reconocimiento de la contradicción entre el deporte escolar y extraescolar, la dificultad de generar nuevas construcciones metodológicas, y la emergencia de componentes eróticos en la comunicación corporal entre varones y mujeres.

La consideración de las diferencias físicas y actitudinales entre varones y mujeres, y el peligro que supone el contacto corporal se sostiene desde una concepción binaria y esencialista de los cuerpos y la sexualidad. La complejidad humana es organizada de manera dicotómica, estableciendo dos formas opuestas y desconectadas para lo masculino y femenino, lo cual es definido sobre la base de la biología que es asumida como "natural". Esta mirada esencialista establece una continuidad lineal entre la biología, la identidad de género y el deseo sexual, desde un modelo cisnormativo y heteronormativo.

Los otros dos argumentos que sostienen el rechazo a las clases mixtas -aquellos que remiten a la visualización de la contradicción entre el deporte escolar y los certámenes deportivos extraescolares y la dificultad para generar propuestas coeducativas- se sostienen desde un enfoque tradicional de la EF, vinculado con lógicas tecnicistas y deportivas propias de los paradigmas que fundamentan el entrenamiento deportivo de alto rendimiento. En ese sentido, tal como plantea Kopelovich, "si se piensa el deporte en la escuela a semejanza del deporte del club, con el objetivo de ganar torneos, la posibilidad de trabajar juntos se dificulta" (2017, p. 107).

Por otra parte, además de sostener estos enfoques tradicionales que dificultan la recepción de nuevos enfoques críticos de la EF, el análisis puso en evidencia un desconocimiento de la perspectiva y el modo de abordaje que propone la Educación Sexual Integral para el área de la Educación Física escolar. El no conocer incide en un posicionamiento adverso a la ESI.

Para avanzar entonces en la transversalización de la ESI en este espacio curricular, se hace necesario deconstruir estos sentidos naturalizados 
en torno al cuerpo y al género, para construir nuevos sentidos que reconozcan al cuerpo inscripto en tramas de sentido y significación, desde el concepto de corporeidad, como unidad psicofísica, espiritual, motora, afectiva, social, intelectual (Grasso, 2001) que incluye esquema e imagen corporal e identidad, y de esta manera se visualice la diversidad de construcciones identitarias posibles. Correrse de la mirada generizada de los cuerpos, permitirá reconocer a cada estudiante en su singularidad, y habilitar espacios para sus singulares e identitarios modos de expresión motriz.

Requiere además revisar el sentido que adquiere la EF en la escuela, para "dejar atrás propuestas pedagógicas reproductoras de modelos de enseñanza de los deportes ajenos a lo escolar, con sistemas elitistas y excluyentes, que privilegian a los más aptos, lesionando el derecho a aprender." (Córdoba, Argentina. Ministerio de Educación de la Provincia de Córdoba, 2011, p. 144) y situar a la EF como práctica social en procura de escenarios solidarios y cooperativos, y donde las prácticas deportivas permitan aprender a todas/os posibilitando la aceptación de lo diverso. Es a partir del movimiento que el sujeto expresa el resultado de una praxis, que es definida también por las/os otras/os y el contexto. Así, la EF en la educación secundaria podrá dar respuesta a intereses diversos, a biografías particulares, descartando caminos únicos y exclusivos, que indican unidireccionalidad y linealidad en los aprendizajes corporales y motrices de las/os estudiantes. Apostamos a una EF que pueda abrir con mayor énfasis el juego a otras actividades corporales y motrices, cuya apropiación sea el resultado de la utilización de recursos sensibles, conscientes y creativos: la expresión corporal, la danza, las representaciones simbólicas; el juego corporal, entre otras. Una EF como campo de vivencia social, donde cobra protagonismo el placer por el encuentro y el movimiento.

Sostenemos que es necesario para ello que la formación del profesorado esté atravesada por enfoques sociocríticos, con fuerte presencia de la didáctica de la EF. Además, generar dispositivos de capacitación en ESI, que contribuyan a desnaturalizar las prácticas tradicionales y hegemónicas de EF y tengan como objetivo posibilitar el resurgimiento de construcciones metodológicas inclusivas y contextualizadas que ofrezcan diversidad motriz, placer por el movimiento y equidad social.

\section{Referencias bibliográficas}

Abramowski, A. (2012). Los afectos docentes en las relaciones pedagógicas: tensiones entre querer y enseñar. Congress of the Latin American Studies Association, San Francisco, California, May 23-26.

Argentina. Programa Nacional de Educación Sexual Integral. Ley N. ${ }^{\circ} 26.150$. Sancionada el 4 de octubre de 2006.

Argentina, Ministerio de Educación de la Nación. (2008). Lineamientos Curriculares para la Educación Sexual Integral. Buenos Aires: Argentina.

Báez, J. (2016). La inclusión de la educación sexual en las políticas públicas de América Latina. Los organismos internacionales y sus formas de 
intervención. Revista Latinoamericana de Educación Comparada 7(9), pp. 70-82.

Boccardi, F. (2010). La sexualidad en la red de los discursos mediáticos. Una lectura de la construcción de la educación sexual en la prensa argentina. Revista F@ro, Año 6, № 12, pp. 10-17. Recuperado de: https://dialnet.u nirioja.es/servlet $/$ articulo?codigo $=3625810$

Boccardi, F. (2014). Acerca de la integralidad. Una lectura semiótica de los fundamentos de la educación sexual integral. En: Amman, B; Boria, A; Da Porta, E.; Maccioni, L. y Uzín, M.: Sujetos emergentes y prácticas culturales: experiencias y debates contemporáneos. Córdoba: Ferreyra Editor, pp. 219-227.

Boccardi, F. (2020). Lo 'sexual' y lo 'reproductivo'. Una genealogía de las definiciones de sexualidad en la arena discursiva internacional de los derechos. KAIROS. Revista de Temas Sociales. Publicación de la Universidad Nacional de San Luís. Año 24. No 46. Diciembre de 2020. en prensa

Bracht, V. (1996). Educación Física y aprendizaje social. Educación Fisica/ciencia del deporte: ¿Qué ciencia es esa? Córdoba: Editorial Vélez Sarsfield.

Bracht, V. \& Crisonio, R. (coord.) (2003). La educación fisica en Argentina y en Brasil: Identidad, desafios y perspectivas. La Plata: $\mathrm{Al}$ margen.

Fernández, M. L. (2011). Los estereotipos de género en las clases de Educación física en el nivel medio (Tesis de Licenciatura). Recuperado el 10 de marzo de 2020 de: http://imgbiblio.vaneduc.edu.ar/fulltext/files/TC104960.p $\mathrm{df}$

Córdoba, Argentina. Ministerio de Educación de la provincia de Córdoba. (2011). Diseño curricular del ciclo básico de la educación secundaria 2011-2015. Recuperado el 16 de febrero de 2020 de: https://www.igualdadycalidadcba.gov.ar/SIPEC-CBA/publicaciones /EducacionSecundaria/LISTO\%20PDF/TOMO\%202\%20Ciclo\%20B asico\%20de\%20la\%20Educacion\%20Secundaria\%20web\%208-2-11.pdf

Di Gregorio, M. J. (2013). ¿Qué enseñamos en Educación Física en relación al género y la sexualidad?: Una propuesta de integración en la educación secundaria [en línea]. 10mo Congreso Argentino de Educación Física y Ciencias, 9 al 13 de septiembre de 2013, La Plata. En Memoria Académica. Disponible en: http://www.memoria.fahce.unlp.edu.ar/trab_eventos/ev. 3199/ev.3199.

Galak, E. (2009). El cuerpo de las prácticas corporales. Educación Física. Estudios Críticos en Educación Física, La Plata: Al Margen pp. 271-284 https://eduardogalak.files.wordpress.com/2010/10/el-cuerpode-las-practicas-corporales-eduardo-galak.pdf

Grasso, A. (2001). El aprendizaje no resuelto de la Educación Física. La corporeidad. Buenos Aires: Novedades Educativas

Giordan, A. \& De Vecchi, G. (1988). Los origenes del saber. Sevilla: Diada

Glaser, B., Strauss, A. (1967). The discovery of grounded theory. New York: Aldine publishing Company

Gómez, J., Teves, N. (2013). La construcción de género en el campo de la educación fisica [en línea]. 10mo Congreso Argentino de Educación Física y Ciencias, 9 al 13 de septiembre de 2013, La Plata. En Memoria Académica. Disponible en: http://www.memoria.fahce.unlp.edu.ar/trab _eventos/ev.3200/ev.3200.pdf 
Gomez, R. (2009). Pedagogía del deporte y reflexividad: elementos para una teoría de la enseñanza del deporte en la escuela latinoamericana en Martínez Álvarez y Gómez (coord) La Educación Física y el deporte escolar. El giro reflexivo en la enseñanza. Buenos Aires: Miño y Dávila

Jenkins, Ph. (1998). Moral panic. Changing concepts of the child molester in modern America. New Haven: Yale University Press.

Jones, D. (2010). Sexualidades adolescentes: amor, placer y control en la Argentina contemporánea. Buenos Aires: CICCUS.

Kopelovich, P. (2017). Educación física y escuela secundaria. Sentidos y prácticas en torno a la enseñanza de la asignatura en un colegio de la provincia de Buenos aires (Argentina). (Tesis de Maestría) Recuperada el 25 de octubre de 2019 de https://repositorio.flacsoandes.edu.ec/bitstream/10469/137 29/2/TFLACSO-2017PK.pdf

López Estevez, R. (2012). La coeducación en el área de Educación Física: revisión, análisis y factores condicionantes. Lecturas: Educación Física y Deportes, Revista Digital. 169. Buenos Aires. Recuperado el 12 de marzo de 2020 de http://file://D:/Documents/Downloads/Dialnet-LaCoedu cacionEnElAreaDeEducacionFisica-4730353.pdf

Margulis, M. (2003). Mandatos culturales sobre la sexualidad y el amor. En: Margulis (comp.): Juventud, cultura, sexualidad. La dimensión cultural en la afectividad y la sexualidad de los jóvenes de Buenos Aires. Buenos Aires: Biblos

Millet, K. (1975). Política sexual. México: Aguilar.

Pellegrini Malpiedi, M. (2015). Pedagogía y educación fisica: prescripciones para un cuerpo hegemónico. Recuperado el 12 de junio de 2019 de: http://reph ip.unr.edu.ar/xmlui/handle/2133/5429.

Pozo, J.I.; Del Puy Pérez, M.; Sanz, A.; Limón, M. (1992). Las ideas de los alumnos sobre la ciencia como teorías implícitas. Infancia y Aprendizaje. $57,3-22$

Sanchez Perez, A. (2012) La coeducación en Educación Física. (Trabajo final de máster). Recuperado el 28 de octubre de 2019 de https://repositorio.unican.es/xmlui/bitstream/handle/10902/1749/ S\%C3\%A1nchez\%20P\%C3\%A9rez\%20\%C3\%81ngela.pdf?sequence=1

Scharagrodsky, P. (2004). Juntos pero no revueltos: la Educación Física mixta en clave de género. Cuadernos de Pesquisa, 34, pp. 59-76.

Scharagrodsky, P. (2006). El padre de la Educación Física argentina: fabricando una política corporal generizada (1901-1938) En A. Aistenstein \& P. Scharagrodsky, Tras las huellas de la Educación física escolar argentina. Cuerpo, género y pedagogia: 1880-1950 (pp.159-197). Buenos Aires: Prometeo.

Serano, J. (2007). Whipping Girl: A Transsexual Woman on Sexism and the Scapegoating of Femininity. Emeryville: Seal Press.

Serra Payeras, P. (2016). La perspectiva de género en los estudios de ciencias de la actividad física y el deporte. (Tesis doctoral) Recuperado el 18 de febrero de 2020 de https://inefcgiseafe.files.wordpress.com/2016/07/pedrona-serra-paye ras_2016_la-perspectiva-de-gc3a9nero-en-los-estudios-de-cafd-tesis-doct oral.pdf

Thompson, K. (2014). Pánicos morales. Bernal: Universidad Nacional de Quilmes Editorial. 
Trueba, S. (2007). Las Concepciones de género en las clases de Educación Física. (Tesina de grado) Universidad Nacional del Litoral) Recuperado el 12 de septiembre de 2018 de https://www.researchgate.net/publication/330094826_Las_Concep ciones_de_genero_en_las_clases_de_Educacion_Fisica_Tesina_de_Lice nciatura_en_Educacion_Fisica_-_Universidad_Nacional_del_Litoral

Vallarino, R. (2017). El criterio de división sexual en la clase de Educación Física. EN: Actas del $12^{\circ}$ Congreso Argentino de Educación Física y Ciencias. Ensenada: Universidad Nacional de La Plata. Facultad de Humanidades y Ciencias de la Educación. Departamento de Educación Física. Disponible en: http://www.memoria.fahce.unlp.edu.ar/trab_eventos/ev.10216/ev.1 0216.pdf

Vance, C. (1997). La antropología redescubre la sexualidad. El Colegio de México, Centro de Estudios Demográficos y de Desarrollo Urbano. Doi: htt p://dx.doi.org/10.24201/edu.v12i1.989

Vázquez y Gomez, B., Manzano, A., Alvarez Bueno, G., Fernández García E, Cano de mateos, S, López, C. (1990). Guía para una educación no sexista. Secretaría General Técnica. Centro de Publicaciones. Ministerio de Educación y Ciencia. España. https://sede.educacion.gob.es/publivent a/guia-para-una-educacion-fisica-no-sexista/sociologia/688

Warner, M. (1991). Fear of a queer planet. Queer politics and Social Theory, University of Minnesota Press: Londres.

\section{Notas}

1 Proyecto de Investigación "El posicionamiento docente frente a la implementación de la Educación Sexual Integral en Educación Física de la escuela secundaria”, Marozzi, J. (directora); Bertarelli, P.; Boccardi, F.; Bosio, M.; Raviolo, A.; Videla, S. UPC 2017-2019. Aprobado y financiado por el Ministerio de Ciencia y Tecnología Córdoba.

2 En el texto se colocan nombres y edades de las/os entrevistadas/os. En caso que sea un enunciado explicitado en un curso de capacitación, se coloca el número de curso.

3 Una de las actividades propuestas durante los cursos de capacitación a docentes, donde se llevó a cabo esta investigación, solicitaba reconstruir clases que dictaban las/os docentes. El 90\% de las clases reconstruidas daban cuenta de la enseñanza de un deporte. 